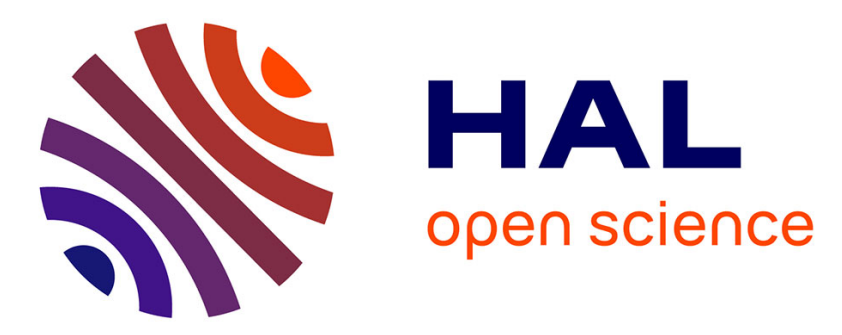

\title{
PEDOT-modified integrated microelectrodes for the detection of ascorbic acid, dopamine and uric acid
}

Fadhila Sekli-Belaidi, Aurélie Civélas, Valentina Castagnola, Aliki Tsopela, Laurent Mazenq, Pierre Gros, Jérôme Launay, Pierre Temple-Boyer

\section{- To cite this version:}

Fadhila Sekli-Belaidi, Aurélie Civélas, Valentina Castagnola, Aliki Tsopela, Laurent Mazenq, et al.. PEDOT-modified integrated microelectrodes for the detection of ascorbic acid, dopamine and uric acid. Sensors and Actuators B: Chemical, 2015, 214, pp. 1-9. 10.1016/j.snb.2015.03.005 . hal-01148710

\author{
HAL Id: hal-01148710 \\ https://hal.science/hal-01148710
}

Submitted on 5 May 2015

HAL is a multi-disciplinary open access archive for the deposit and dissemination of scientific research documents, whether they are published or not. The documents may come from teaching and research institutions in France or abroad, or from public or private research centers.
L'archive ouverte pluridisciplinaire HAL, est destinée au dépôt et à la diffusion de documents scientifiques de niveau recherche, publiés ou non, émanant des établissements d'enseignement et de recherche français ou étrangers, des laboratoires publics ou privés. 


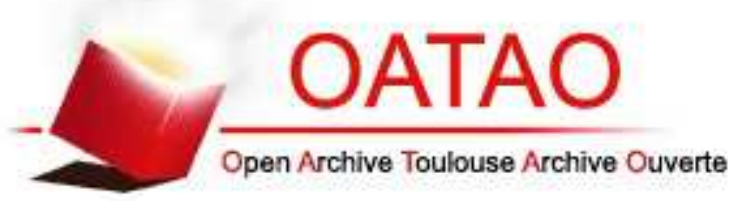

\section{Open Archive TOULOUSE Archive Ouverte (OATAO)}

OATAO is an open access repository that collects the work of Toulouse researchers and makes it freely available over the web where possible.

This is an author-deposited version published in : http://oatao.univ-toulouse.fr/ Eprints ID : 13875

To link to this article : DOI:10.1016/j.snb.2015.03.005

URL : http://dx.doi.org/10.1016/j.snb.2015.03.005

\section{To cite this version :}

Sekli-Belaidi, Fadhila and Civélas, Aurélie and Castagnola, Valentina and Tsopela, Aliki and Mazenq, Laurent and Gros, Pierre and Launay, Jérôme and Temple-Boyer, Pierre PEDOT-modified integrated microelectrodes for the detection of ascorbic acid, dopamine and uric acid. (2015) Sensors and Actuators B: Chemical, 214. pp. 1-9. ISSN 0925-4005

Any correspondance concerning this service should be sent to the repository administrator: staff-oatao@ listes-diff.inp-toulouse.fr 


\title{
PEDOT-modified integrated microelectrodes for the detection of ascorbic acid, dopamine and uric acid
}

\author{
F. Sekli Belaidi ${ }^{a, b, c, d}$, A. Civélas ${ }^{a, b}$, V. Castagnola ${ }^{a, b}$, A. Tsopela ${ }^{a, b}$, L. Mazenq $^{\mathrm{a}, \mathrm{b}}$, \\ P. Gros ${ }^{c, d}$, J. Launay ${ }^{\mathrm{a}, \mathrm{b}}$, P. Temple-Boyer ${ }^{\mathrm{a}, \mathrm{b}, *}$ \\ a CNRS, LAAS, 7 avenue du colonel Roche, F-31400 Toulouse, France \\ ${ }^{\mathrm{b}}$ Université de Toulouse, UPS, LAAS, F-31400 Toulouse, France \\ c Université de Toulouse, UPS, INPT, LGC, F-31062 Toulouse, France \\ d CNRS, LGC, F-31062 Toulouse, France
}

Keywords:

Integrated microelectrode

Electrochemical microcell

PEDOT

Ascorbic acid

Dopamine

Uric acid

\begin{abstract}
A B S T R A C T
Integrated (Pt/PEDOT-Pt-Ag/AgCl) and (Au/PEDOT-Pt-Ag/AgCl) electrochemical microcells (ElecCell) were elaborated for the detection of ascorbic acid, dopamine and uric acid by differential pulse voltammetry. Specific attention was brought to the integration of poly(3,4-ethylenedioxythiophene) (PEDOT) film by electropolymerization. Gold and platinum working microelectrodes were investigated while using ethylenedioxythiophene (EDOT) electrodeposition processes in water or acetonitrile solvents. For the three antioxidant species, best (multi-)detection properties were obtained for acetonitrile-based PEDOT films deposited on gold working electrode. Thus, using integrated (Au/PEDOT-Pt-Ag/AgCl) ElecCell microdevices, analytical performances were determined for ascorbic acid, dopamine and uric acid, exhibiting high selectivity (oxidation potential: $-40,150$ and $280 \mathrm{mV}$, respectively), linear concentration range from 0.1 to $300 \mu \mathrm{M}$, high sensitivities $\left(0.85,1.65\right.$ and $3.06 \mu \mathrm{A} / \mu \mathrm{M} \mathrm{cm}^{2}$, respectively) and low detection limit $(0.2 \mu \mathrm{M}, 0.1 \mu \mathrm{M}$ and $0.05 \mu \mathrm{M}$, respectively).
\end{abstract}

\section{Introduction}

During the last two decades, the area of sensors has greatly benefited from the development of micro/nanotechnologies in term of design, fabrication and detection performances. This was also true for chemical microsensors and electrochemical analysis for biosensing applications. Consequently, integrated microelectrodes have become well-accepted tools for clinical, environmental, chemical and pharmaceutical applications with high spatial and temporal resolution [1,2]. Indeed, they present many advantages: specificity, high sensitivity, fast response time, small capacitive currents, enhanced mass transport, low ohmic drop allowing their use in low conducting and highly viscous media, as well as versatility. Moreover, compared to ultra-microelectrodes (UME) sealed into glass-capillaries [3-6], they take advantage of mass fabrication at low cost thanks to the use of silicon-based microtechnologies [7-9], addressing many bioanalytical applications [10-14]. Nevertheless, to realize a simple and functional electrochemical sensor,

\footnotetext{
* Corresponding author at: CNRS, LAAS, 7 avenue du colonel Roche, F-31400 Toulouse, France. Tel.: +33 561336954

E-mail address: temple@laas.fr (P. Temple-Boyer).
}

microfabrication strategies have to address the problems related to the analysis of real samples, emphasizing on sensitivity, selectivity, stability, reproducibility and reliability. We have selected this approach to develop an integrated electrochemical microsensor for the simultaneous detection of ascorbic acid (AA), dopamine (Dop) and uric acid (UA) in the frame of antioxidant species analysis.

The detection of these three analytes is of particular interest in clinical, chemical, pathology, food analysis and many other fields [15-17]. AA is a vital vitamin popularly known for its antioxidant properties and is present in mammalian brain along with several neurotransmitter amines such as dopamine. Ascorbic acid has been used for prevention and treatment of common cold, mental illness, infertility and cancer [18]. Dopamine is an important neurotransmitter for message transfer in central nervous system [19]. Abnormal levels of Dop lead to neurological disorders such as Parkinsonism and Schizophrenia [20]. Meanwhile, uric acid is the primary final product of purine metabolism. The extreme abnormalities of UA levels lead to some diseases, such as hypertension, hyperuricaemia, gout and Lesch-Nyan diseases [21].

In real biological samples, AA, Dop and UA usually coexist, so the development of accurate, selective and simultaneous determination methods for these three analytes is highly desired especially in biomedical chemistry and medical diagnostics. AA, Dop and UA 
are electroactive compounds and can be detected using electroanalytical techniques. Unfortunately, with bare unmodified metallic electrodes, they are oxidized at nearly same potentials and their voltammetric responses overlap makes their discrimination in real samples very difficult $[22,23]$. Besides, bare electrodes often suffer from a pronounced fouling affect due to the accumulation of oxidized products on electrode surface. Furthermore, the modified electrode must be insensitive to interfering chemicals present in biological media. To overcome this problem, many modification strategies have been adopted to lower the overpotential, to increase detection sensitivity and to improve selectivity. In the frame of antioxidant detection, they have led to the realization of various modified (micro)electrodes based on quantum dots [24], nanoparticles [25-27], carbon nanotubes [28-30], graphene [25-27,29,31-33] and conductive polymers [28,34-36]. Among them, poly(3,4-ethylenedioxythiophene) (PEDOT) was one of the widely used conducting polymers for the detection of AA, Dop and UA [37-42]. It has a low oxidation potential and moderate band gap with good stability and transparency in the oxidized state, high electrical conductivity [43], excellent thermal stability, intrinsically low thermal conductivity and low price $[44,45]$. In parallel, electropolymerization is one of the methods used for the preparation of polymer film with good quality. It allows the reproducible formation of organic polymer films with precise spatial resolution. Moreover, film thicknesses are easily controlled by the deposition charge and the polymer is directly obtained in his conducting state [46]. Thus, electrodeposition protocol of PEDOT is easier compared to others strategies of electrode modifications. Finally, ethylenedioxythiophene (EDOT) is a commercially available monomer that eliminates synthesis steps.

In the frame of the detection of antioxidant species, PEDOT acts as a redox mediator responsible for oxidation catalysis. Since ascorbic and uric acids are in their anionic form $\left(\mathrm{HA}^{-}\right)$at physiological $\mathrm{pH}$, the occurring catalytic mechanism is globally given by [47]:

PEDOT $_{\mathrm{ox}}+\mathrm{HA}^{-} \rightarrow$ PEDOT $_{\mathrm{red}}{ }^{-}+\mathrm{A}$

PEDOT $_{\text {red }}{ }^{-} \rightarrow$ PEDOT $_{\text {ox }}+\mathrm{H}^{+}+2 \mathrm{e}^{-}$

In the case of dopamin known to be in its cationic form at physiological pH, the global catalytic mechanism is [37]:

$\mathrm{PEDOT}_{\mathrm{ox}}+\mathrm{Dop} \rightarrow$ PEDOT $_{\text {red }}+\mathrm{DoQ}$

PEDOT $_{\text {red }} \rightarrow$ PEDOT $_{\mathrm{ox}}+2 \mathrm{H}^{+}+2 \mathrm{e}^{-}$

Our previous works illustrated that PEDOT deposited on hand-made microelectrodes has good catalytic properties for the electrochemical oxidation of ascorbic and uric acids and can be used for their simultaneous detection [39]. This work goes further towards technological integration and mass fabrication of PEDOTbased microelectrodes, focusing on three main goals: (i) to study the electropolymerization of PEDOT on thin-film-based microelectrodes, (ii) to integrate fully PEDOT-based electrochemical microcells (ElecCell), and (iii) to analyze PEDOT-based ElecCell performances for the selective detection of antioxidant species. Thus, combining the advantageous features of silicon-based microtechnologies [23,48] and catalytic properties of PEDOT [46], we presented here the analytic performances of integrated electrochemical microdevices modified with PEDOT electrodeposited in different conditions of polymerization for a simultaneous assay of AA, Dop, and UA.

\section{Experimental}

\subsection{Chemicals}

3,4-Ethylenedioxythiophene (EDOT) monomer, poly(sodium 4styrenesulfonate) (NaPSS), ascorbic acid (AA), dopamine (Dop) and uric acid (UA) were purchased from Sigma Aldrich. Tetrabutylammonium perchlorate (TBAPC), potassium dihydrogenophosphate $\mathrm{KH}_{2} \mathrm{PO}_{4}$, di-potassium hydrogenophosphate $\mathrm{K}_{2} \mathrm{PHO}_{4}$, sodium chloride $\mathrm{NaCl}$ and acetonitrile $\mathrm{CH}_{3} \mathrm{CN}$ were purchased from Acros. All reagents were of analytical grade and used as received. The aqueous solutions were prepared with high-quality water (MilliQ gradient A10 system, Millipore, Bedford, MA). High pure nitrogen was used for deaeration.

\subsection{Materials}

Electrochemical Impedance Spectroscopy (EIS) measurements were made in $0.1 \mathrm{M} \mathrm{NaCl}$ solution by applying a $5 \mathrm{mV}$ RMS sine wave with frequencies ranging from $10 \mathrm{~Hz}$ to $10 \mathrm{kHz}$. Scanning electron microscopy (SEM) studies were carried out using a focused ion beam (FIB) HELIOS 600i equipment operating at $3 \mathrm{kV}$. Samples were mounted on a double-sided adhesive carbon and optical microscope images were then made using a Hirox Microscope (HI-SCOPE advanced KH-3000). PEDOT electropolymerization and electrochemical experiments were performed using a VMP3 potentiostat (Biologic) interfaced to a microcomputer and using the EC-Lab software.

\subsection{Electrochemical microcell (ElecCell) fabrication}

Integrated (Pt-Pt- $\mathrm{Ag} / \mathrm{AgCl})$ and $(\mathrm{Au}-\mathrm{Pt}-\mathrm{Ag} / \mathrm{AgCl})$ electrochemical microcells (ElecCell) were fabricated on silicon chip using silicon-based microtechnologies (Fig. 1a) [23]. Oxidized silicon wafers were used in order to ensure electrical insulation between the different microelectrodes (oxide thickness: $\sim 1 \mu \mathrm{m}$ ). Then, the different thin metallic layers were deposited by evaporation in conventional physical vapour deposition (PVD) equipment, and patterned using a bilayer lift-off process in order to improve fabrication reproducibility. Three PVD processes were performed in a row: firstly, a $200 \mathrm{~nm}$ platinum layer was deposited on a $20 \mathrm{~nm}$ titanium underlayer in order to ensure platinum adhesion on silicon oxide, followed by a $800 \mathrm{~nm}$ gold and a $400 \mathrm{~nm}$ silver layers. Finally, a biocompatible $\mathrm{Si}_{3} \mathrm{~N}_{4}$ passivation layer (thickness: $100 \mathrm{~nm}$ ) was deposited at the wafer level and patterned using photolithography techniques [48]. According to this final wafer-level passivation process, the different metallic layers were insulated electrically and their active surfaces were defined precisely. The gold and platinum working microelectrodes were defined as disks and their electroactive area was approximately $4.9 \times 10^{-4} \mathrm{~mm}^{2}$ (diameter: $25 \mu \mathrm{m}$ ). In contrast, very large silver/silver chloride reference microelectrode $\left(0.02 \mathrm{~mm}^{2}\right)$ and platinum counter microelectrode $\left(1 \mathrm{~mm}^{2}\right)$ were fabricated. After the silicon wafer dicing, (Pt-Pt-Ag) and (Au-Pt-Ag) electrochemical microcells were manufactured on silicon chip (Fig. 1a). The whole chip was then placed and glued by an epoxy insulating glue on a specifically coated printed circuit, wire bonded and packaged at the system level using a silicone glop-top in order to be fully compatible with liquid phase measurement.

For each microdevice, the silver/silver chloride $\mathrm{Ag} / \mathrm{AgCl}$ pseudoreference was finally obtained by oxidizing the silver-based microelectrode in a $0.01 \mathrm{M} \mathrm{KCl}$ solution. This was performed by linear voltammetry (potential scan rate: $1 \mathrm{mV} / \mathrm{s}$ between 0.1 and $0.25 \mathrm{~V} / \mathrm{SCE}$ ) using a standard saturated calomel electrode (SCE) $\mathrm{Hg} / \mathrm{Hg}_{2} \mathrm{Cl}_{2} / \mathrm{KCl}_{\text {sat }}$ as reference. Thus, (Pt-Pt-Ag/AgCl) and ( $\mathrm{Au}-\mathrm{Pt}-\mathrm{Ag} / \mathrm{AgCl}$ ) ElecCell microdevices were finally realized. 

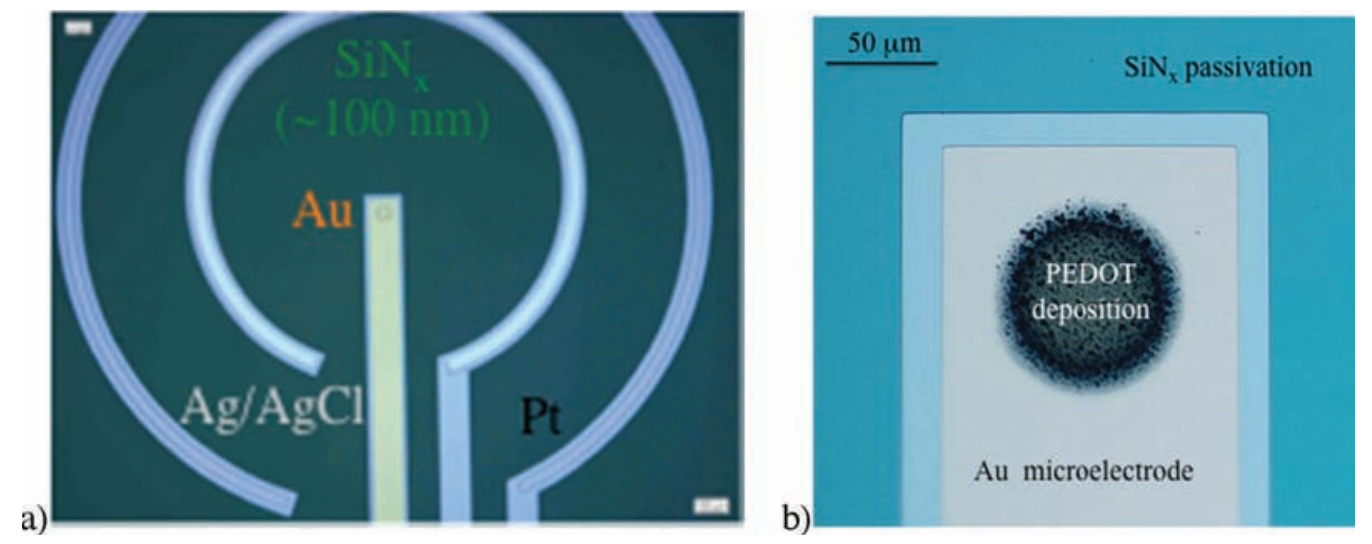

Fig. 1. Optical microscope images of (a) the integrated (Au-Pt-Ag/AgCl) electrochemical microcell (ElecCell) device and (b) the electrodeposited PEDOT film on the gold working electrode (solvent: acetonitrile)

\subsection{Preparation and characterization of PEDOT modified electrode}

PEDOT electropolymerization processes were carried out in organic, i.e. acetonitrile-based, or inorganic, i.e. water-based, solutions.

For the organic acetonitrile-based process, the integrated working microelectrode surface was modified in a deaerated acetonitrile solution containing $2.5 \mathrm{mM}$ EDOT monomer and $0.1 \mathrm{M}$ TBAPC as supporting electrolyte [39]. Then, polymerization was performed by cyclic voltammetry at a scan rate of $250 \mathrm{mV} / \mathrm{s}$ between 0.88 and $1.5 \mathrm{~V}$.

For the inorganic water-based process, electropolymerization experiment was performed from EDOT $(0.1 \% \mathrm{~W} / \mathrm{V}, 0.01 \mathrm{M})$ and NaPSS $(0.7 \% \mathrm{~W} / \mathrm{V})$ in aqueous deaerated solutions. Such concentration was lower than EDOT solubility in water (estimated around $15 \mathrm{mM}$ at $25^{\circ} \mathrm{C}$ ) to ensure its complete dissolving. Then, cyclic voltammetry was carried between -0.9 and $1.2 \mathrm{~V}$ at a scan rate of $25 \mathrm{mV} / \mathrm{s}$ [49].

In both cases, i.e. acetonitrile or water solvents, the amount of PEDOT synthesized corresponded to the same anodic charge of $12 \mathrm{mC} / \mathrm{cm}^{2}$. After the electropolymerization, the modified electrodes were rinsed with acetonitrile and/or deionized water in a row to remove any physically adsorbed monomer (Fig. 1b).

\subsection{Electrochemical experiments of PEDOT-based ElecCell integrated microdevice}

For the quantitative determination of AA, Dop and UA, differential pulse voltammetry (DPV) was investigated since it is more sensitive than cyclic voltammetry. Differential pulse voltammograms were collected in the potential range between 0.2 and $0.4 \mathrm{~V}$, with a $50 \mathrm{mV}$ amplitude, a $6 \mathrm{mV}$ potential step, a $119 \mathrm{~ms}$ pulse time, a $1 \mathrm{~s}$ interval time and a $6 \mathrm{mV} / \mathrm{s}$ potential scan rate. Integrated (Au/PEDOT-Pt-Ag/AgCl) and (Pt/PEDOT-Pt-Ag/AgCl) electrochemical microcells were used for these DPV experiments. For each of them, gold or platinum PEDOT-modified microelectrodes were used as working electrodes whereas the platinum and silver/silver chloride microelectrodes were used as counter and pseudo-reference electrodes, respectively. All electrochemical experiments were performed in a glass cell containing $100 \mathrm{~mL}$ of $0.1 \mathrm{M}$ deaerated phosphate buffer solution ( $\mathrm{PBS}, \mathrm{pH}=7.0$ ) with different concentrations of AA, Dop, and UA. The standard addition method was applied for drawing the calibration curves for each specie. Freshly concentrated solutions of AA, Dop, and UA were prepared and stored at $4{ }^{\circ} \mathrm{C}$. Then a small known concentration of desired element is increasingly added to PBS solutions. Currents were then plotted against the added concentrations. The limit of detection was estimated for a signal-to noise-ratio equal to three.

\section{Results and discussion}

\subsection{Effect of EDOT solvent}

The solvent used during the electropolymerization step has a key influence on the conducting polymers ultimate properties. It should lead to a high electrical conductivity and good electrochemical stability against decomposition at high potentials required to oxidize the monomer. Thus, electrosynthesis of PEDOT is often performed in organic solvent [37,39]. Nevertheless, even if water has some drawbacks such as high nucleophilicity, narrow potential window for electrochemical stability and high EDOT oxidation potential (higher than the acetonitrile one), it was also used as solvent for PEDOT electrodeposition even if the EDOT monomer is slightly soluble in aqueous solution $[49,50]$. Above all these problems, the selection of water as the synthesis medium would be self-evident merely from environmental, economic and biocompatibility reasons.

Fig. 2a and b shows the cyclic voltammograms recorded during PEDOT electrogeneration on a gold integrated microelectrode, in water-based or in acetonitrile-based solutions, respectively. Similar electrochemical behaviours were observed for both solvents. In water (Fig. 2a), the EDOT monomer oxidation starts at $0.6 \mathrm{~V}$ and the anodic current increases from cycle to cycle indicating the polymer growth. Then, the PEDOT redox properties are evidenced at $-0.1 \mathrm{~V}$. The electropolymerization potential decrease was attributed to the strong electrostatic interactions between EDOT $^{\bullet+}$ cation radicals and PSS $^{-}$species, facilitating the polymerization process [51].

In acetonitrile (Fig. 2b), it is clearly visible that the EDOT monomer oxidation stars at $1.2 \mathrm{~V}$ whereas the redox potential of PEDOT is obtained around $-0.25 \mathrm{~V}$. It is known that peaks position of the polymer redox activity is relative to p-doping process, leads to differences in conductivity properties [52], and might indicate that a higher molecular mass polymer is obtained when electrosynthesis is performed in organic medium. Thus, even if a similar anodic charge of $12 \mathrm{mC} / \mathrm{cm}^{2}$ was chosen for the PEDOT synthesis, this should also be responsible for some thickness and morphology discrepancies for the different PEDOT layers.

To have further information on PEDOT depositions, they were characterized by impedancemetry and scanning electron microscopy (SEM). Compared to water solvent, acetonitrile leads to lower impedance modulus and therefore to higher electrical conductivity (data not shown). Such difference in term of electrical conductivity might be explained by the doping level of each PEDOT 



b)

Fig. 2. Cyclic voltammogramms of electropolymerization at gold working microelectrode in deaerated $0.1 \mathrm{~mol} / \mathrm{L}$ TBAPC and $2.5 \mathrm{mmol} / \mathrm{L}$ EDOT (a) water-based (potential scan rate: $25 \mathrm{mV} / \mathrm{s}$ ) and (b) acetonitrile-based solutions (potential scan rate: $250 \mathrm{mV} / \mathrm{s}$ ).

film. Indeed, the use of TBAPC, and especially the perchlorate ion $\mathrm{ClO}_{4}{ }^{-}$, as charge compensation was shown to give PEDOT films with higher doping level and better stability [53].

Nevertheless, more significant results were obtained by SEM. Fig. 3a and $\mathrm{b}$ presents the different surface morphologies of PEDOT layers electrodeposited on gold microelectrode while using water and acetonitrile solvents. In contrast to water-based PEDOT that forms a cauliflower-type, compact structure, acetonitrilebased ones show a porous complex structure. To the best of our knowledge, the effects of solvent on morphological features, and the correlation between the morphology of electropolymerized films and their catalytic properties were never systematically investigated. To explain the significant differences between the morphological properties of PEDOT films prepared in water or in acetonitrile, we can speculate that these changes are attributed to the different intrinsic properties of each solvent that contribute to different solute-solvent and/or polymer-solvent interactions. The best solvents were found to have high dipole moments, low polarizability and high capacity to donate electrons [54]. Furthermore, higher dielectric constants ( $\sim 80$ for water compared to $\sim 36$ for acetonitrile) lead to lower electropolymerization rate and to more compact films [55]. Meanwhile, we cannot exclude the factor that the solubility of EDOT oligomers produced at initial stages of electropolymerization in both solvents might be responsible of such morphological structures [56]. Certainly, in the very beginning stage of polymerization, oxidation of monomers and coupling of radical cations take place. When the chain length of oligomers is high enough, they precipitate onto the electrode, generating the first polymer nuclei. At this point, the PEDOT deposition on the electrode starts, i.e. nucleation begins, and subsequently the propagation of polymer chains and polymer precipitation are the main processes. In water, the presence of poly-styrenesulphonate (PSS), which is a good solubilizing agent for both EDOT monomer and PEDOT polymer, facilitates the formation of relatively long polymeric chains on solution and consequently smoother films are observed. In acetonitrile, short oligomers are deposited on the electrode, leading to a high number of nucleation centres, which yield to more heterogeneous and very rough films as observed in SEM. Finally, since it was shown that the surface morphology is influenced by the polymerization potential [57], electropolymerization at higher oxidation potential $(1.2-1.5 \mathrm{~V})$ in acetonitrile should produces rougher PEDOT films.

The modified microdevices were therefore tested in an equimolar solution of AA, Dop and UA $1 \mathrm{mmol} / \mathrm{L} \mathrm{pH} \mathrm{7.0.} \mathrm{Results} \mathrm{are} \mathrm{shown}$ in Fig. 4. It is clear that the PEDOT grown in acetonitrile has much better performances than the PEDOT grown in water. For acetonitrile-based PEDOT layers, the oxidation peaks of AA, Dop and UA appear at $-0.04,0.15$ and $0.28 \mathrm{~V}$, respectively, and higher sensitivities are evidenced. For water-based ones, oxidation of AA, Dop and UA occurs at more positive potentials, i.e. 0.125, 0.335 and $0.45 \mathrm{~V}$, inducing lower sensitivities. Such results should be associated to the differences between PEDOT films in terms of structure, morphology and electrical conductivity (as shown by SEM and impedancemetric characterizations, see below). In the case of acetonitrile, rougher and more porous morphologies as well as higher electrical conductivity provide larger electroactive surface, faster diffusion phenomena in and out the polymer network, and better access to electroactive sites, enhancing PEDOT films a)

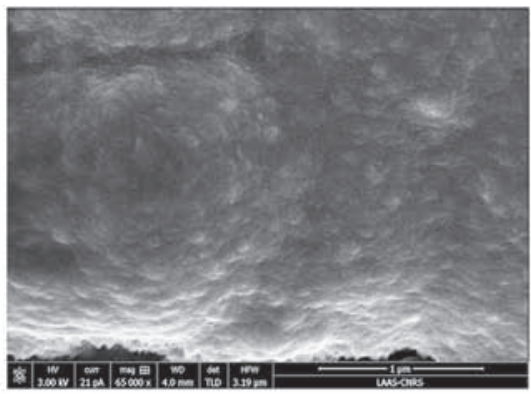

b)

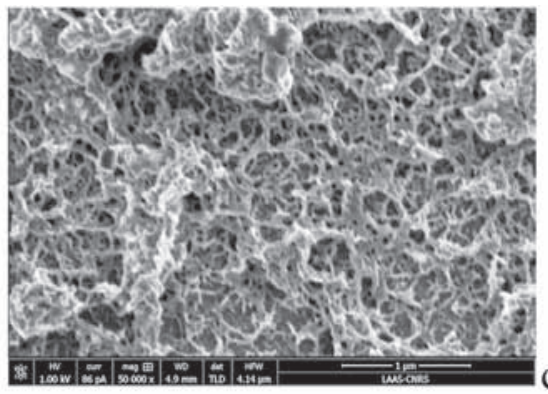

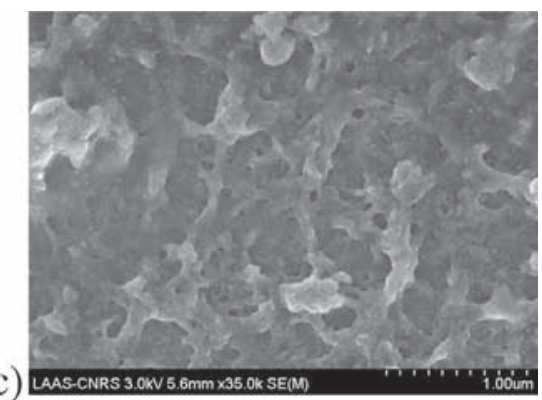

Fig. 3. Scanning electron microscopy (SEM) pictures of PEDOT films deposited on (a) on gold surface using water as solvent, (b) on gold surface using acetonitrile as solvent and (c) on platinum surface while using acetonitrile as solvent 




Fig. 4. Differential pulse voltammograms (DPV) of (Au/PEDOT-Pt-Ag/AgCl) ElecCell in 0.1 M PBS pH 7.0 solution containing an equimolar AA/Dop/UA ( $1 \mathrm{mmol} / \mathrm{L})$ : PEDOT electrodeposited in acetonitrile solution (plain line) or in aqueous solution (dashed line).



Fig. 5. Differential pulse voltammograms (DPV) of (Au-Pt-Ag/AgCl) (plain line) and (Pt-Pt-Ag/AgCl) (dashed line) ElecCell in $0.1 \mathrm{M}$ PBS pH 7.0 solution containing an equimolar AA/Dop/UA mixture $(1 \mathrm{mmol} / \mathrm{L})$.

electrocatalytic properties and improving further antioxidant detection properties [47].

Finally, even if water was successfully developed and gave acceptable results, acetonitrile appears to be the best solvent for integrating PEDOT-modified electrochemical microsensors and

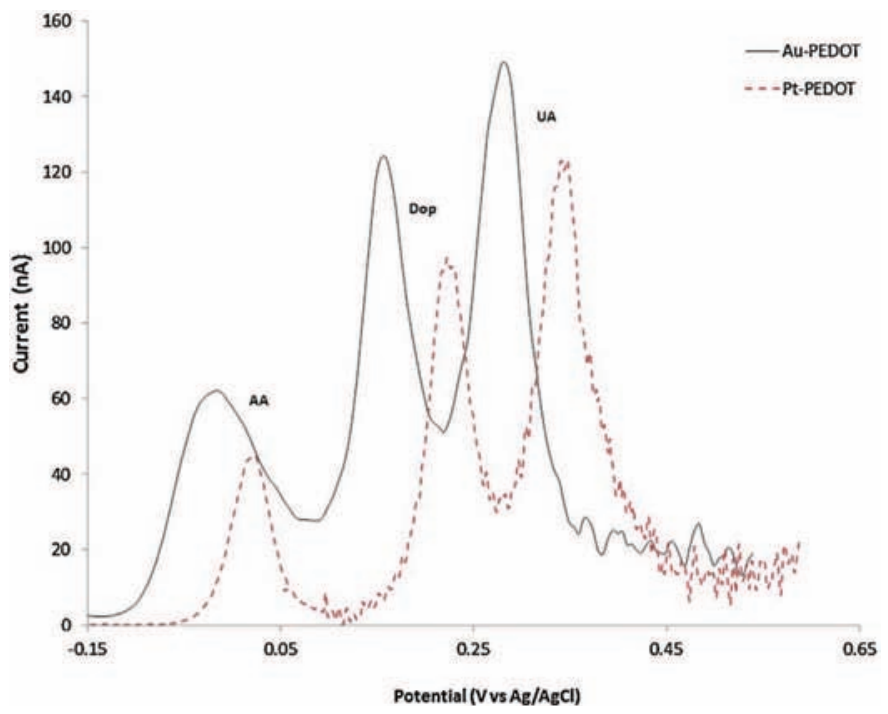

Fig. 6. Differential pulse voltammograms (DPV) of (Au/PEDOT-Pt-Ag/AgCl) (plain line) and (Pt/PEDOT-Pt-Ag/AgCl) (dashed line) ElecCell in $0.1 \mathrm{M} \mathrm{PBS} \mathrm{pH} 7.0$ solution containing an equimolar AA/Dop/UA mixture $(1 \mathrm{mmol} / \mathrm{L})$.

improving PEDOT-based detection performances of antioxidant species in terms of sensitivity and selectivity.

\subsection{Effect of working electrode nature}

As described previously in section 2.3 , the integrated working microelectrode can be made from platinum or gold. Previous works showed that the physico-chemical properties of the anode metallic material could determine the nature and the strength of the bond between the electropolymerized polymer and the electrode, impacting its resulting properties [46]. So, we studied the influence of the metal nature on the PEDOT-based detection properties. In this view, acetonitrile solvent was used for the electrodeposition of PEDOT films on gold and platinum working surfaces (see section 3.1). Then, the electrochemical performances of the PEDOT-modified working microelectrodes were evaluated in an equimolar solution of AA, Dop and UA $1 \mathrm{mmol} / \mathrm{L} \mathrm{pH} \mathrm{7.0.}$ For comparison, bare gold and platinum integrated microelectrodes were also studied in the same way. Results are shown in Figs. 5 and 6.

For bare gold and platinum microelectrodes, a badly defined peak and low current values are observed (Fig. 5). Such amperometric responses were related to competitive oxidation phenomena between AA, Dop and UA. Indeed, by studying separately each analyte (result not shown), their respective oxidation potentials appear at $0.26,0.42$ and $0.47 \mathrm{~V}$ on gold microelectrode, and at $0.32,0.28$ and $0.52 \mathrm{~V}$ on platinum microelectrode, in agreement with previous results [22,23].

On PEDOT-based microelectrodes made from gold or platinum, three well-defined oxidation peaks are observed corresponding to

Table 1

Comparison of the analytical performances of different electrochemical, PEDOT-modified, electrodes for the simultaneous detection of AA, Dop, and UA.

\begin{tabular}{|c|c|c|c|c|c|c|c|c|c|}
\hline \multirow[t]{2}{*}{ Ref. } & \multicolumn{3}{|c|}{$E_{\mathrm{p}} \mathrm{vs} \mathrm{SCE}(\mathrm{mV})$} & \multicolumn{3}{|c|}{ Limit of detection $(\mu \mathrm{M})$} & \multicolumn{3}{|c|}{ Linear range $(\mu \mathrm{M})$} \\
\hline & $\mathrm{AA}$ & Dop & UA & $\mathrm{AA}$ & Dop & UA & $\mathrm{AA}$ & Dop & UA \\
\hline [37] & -50 & 150 & 365 & - & 1 & 1 & - & $1-30$ & $1-20$ \\
\hline [38] & -80 & 120 & 275 & 7.4 & - & - & $500-3500$ & $20-80$ & $20-130$ \\
\hline [39] & -94 & - & 308 & 2.5 & - & 1.5 & $5-300$ & - & $2-600$ \\
\hline [40] & 100 & 250 & 320 & - & - & - & $100-500$ & $100-500$ & $100-500$ \\
\hline [41] & 3 & 210 & 360 & 10 & 1.5 & 2.7 & $20-1400$ & $12-48$ & $36-216$ \\
\hline [42] & 69 & 232 & 364 & 400 & 6 & 2 & $400-8000$ & $6-75$ & $2-40$ \\
\hline This work & -40 & 150 & 280 & 0.2 & 0.1 & 0.05 & $0.5-300$ & $0.2-300$ & $0.1-300$ \\
\hline
\end{tabular}


the oxidation of AA, Dop and UA, respectively (Fig. 6). Compared to the broad and overlapped amperometric responses obtained with bare electrodes, all above results clearly validate the catalytic activity of PEDOT for the electrochemical oxidation of AA, Dop and UA by lowering the oxidation potential and increasing the current $[37,38,47]$. Nevertheless, electrochemical performances are still slightly lower on platinum PEDOT modified microelectrode: the peak potentials are shifted to more positive values, and more precisely at $0.01,0.215$ and $0.34 \mathrm{~V}$, respectively (compared to -0.04 , 0.15 and $0.28 \mathrm{~V}$, see section 3.1), and with lower sensitivities. Earlier, by studying the experimental conditions of polymerization, we have observed that the morphological properties of PEDOT films determine to a large extent the catalytic behaviour for the assay of AA and UA [39]. So, this electrochemical performances discrepancy could be also due to the electrical, morphological and structural properties of the resulting polymers. Indeed, through impedancemetric characterization, PEDOT synthesized on gold is confirmed to have the higher electrical conductivity compared to platinum one. These differences can be due either to the intrinsic conductivities or to the roughness of PEDOT films [49]. Furthermore, SEM characterizations show that acetonitrile-based PEDOT films deposited on platinum surface show less porous structure than those deposited on gold surface (Fig. 3b and c). On the other hand, PEDOT adhesion is best on gold surface due to the strong interactions between gold and sulphur atoms $[58,59]$. Thus, compared to platinum-based ones, (Au/PEDOT-Pt-Ag/AgCl) ElecCell integrated microdevices are more suitable for the simultaneous electrochemical determination of antioxidant species at millimolar concentration levels.

\subsection{Analytical performances}

According to our previous results and optimizations (see sections 3.1 and 3.2), acetonitrile-based PEDOT electrodeposition was performed on gold microelectrode. Since silicon-based integration enables mass fabrication, these investigations were performed for five different $(\mathrm{Au} / \mathrm{PEDOT}-\mathrm{Pt}-\mathrm{Ag} / \mathrm{AgCl})$ electrochemical microcells. Fig. 7 a $-c$ represents the DPV responses of the PEDOT-modified microelectrodes to various concentrations of AA, Dop and UA, respectively. Calibration plots indicate an excellent linearity of the amperometric responses with AA, Dop and UA concentrations at $-0.04,0.15$ and $0.28 \mathrm{~V}$, respectively (Fig. 8). For AA, an excellent linear relationship (sensitivity: $0.85 \mu \mathrm{A} / \mu \mathrm{M} \mathrm{cm}^{2}$ ) was obtained in the concentration range from 0.5 to $300 \mu \mathrm{M}$, with a limit of detection estimated at $0.2 \mu \mathrm{M}$ for a signal to noise ratio of 3. Then, the calibration for dopamin was also found to be linear in the range of $0.2-300 \mu \mathrm{M}$. In this case, a higher slope $\left(1.65 \mu \mathrm{A} / \mu \mathrm{M} \mathrm{cm}^{2}\right)$ value and a limit of detection of $0.1 \mu \mathrm{M}$ were evidenced. Finally, in the case of UA, a linear relationship was found again in the range of $0.1-300 \mu \mathrm{M}$ with a still higher sensitivity $\left(3.06 \mu \mathrm{A} / \mu \mathrm{M} \mathrm{cm}^{2}\right)$ and a limit of detection of $0.05 \mu \mathrm{M}$. All these analytical responses can be resumed as following $\left(R^{2}>0.998\right)$ :

Ascorbic acid detection (oxidation potential: $-0.04 \mathrm{~V}$ ): $j\left(\mu \mathrm{A} / \mathrm{cm}^{2}\right)=24+0.85 C_{\mathrm{AA}}(\mu \mathrm{M})$;

Dopamine detection (oxidation potential: $0.15 \mathrm{~V}$ ): $j\left(\mu \mathrm{A} / \mathrm{cm}^{2}\right)=9.7+1.65 C_{\text {Dop }}(\mu \mathrm{M})$;

Uric acid detection (oxidation potential: $0.28 \mathrm{~V}$ ): $j\left(\mu \mathrm{A} / \mathrm{cm}^{2}\right)=25+3.06 C_{\mathrm{UA}}(\mu \mathrm{M})$.

In term of concentration ranges, these results were well suited to the assay of these analytes in medical fields [60,61]. Compared to works reported in literature for the simultaneous determination of AA, Dop, and UA on PEDOT-modified electrodes, it is worth to note that our results were better or comparable to most of these
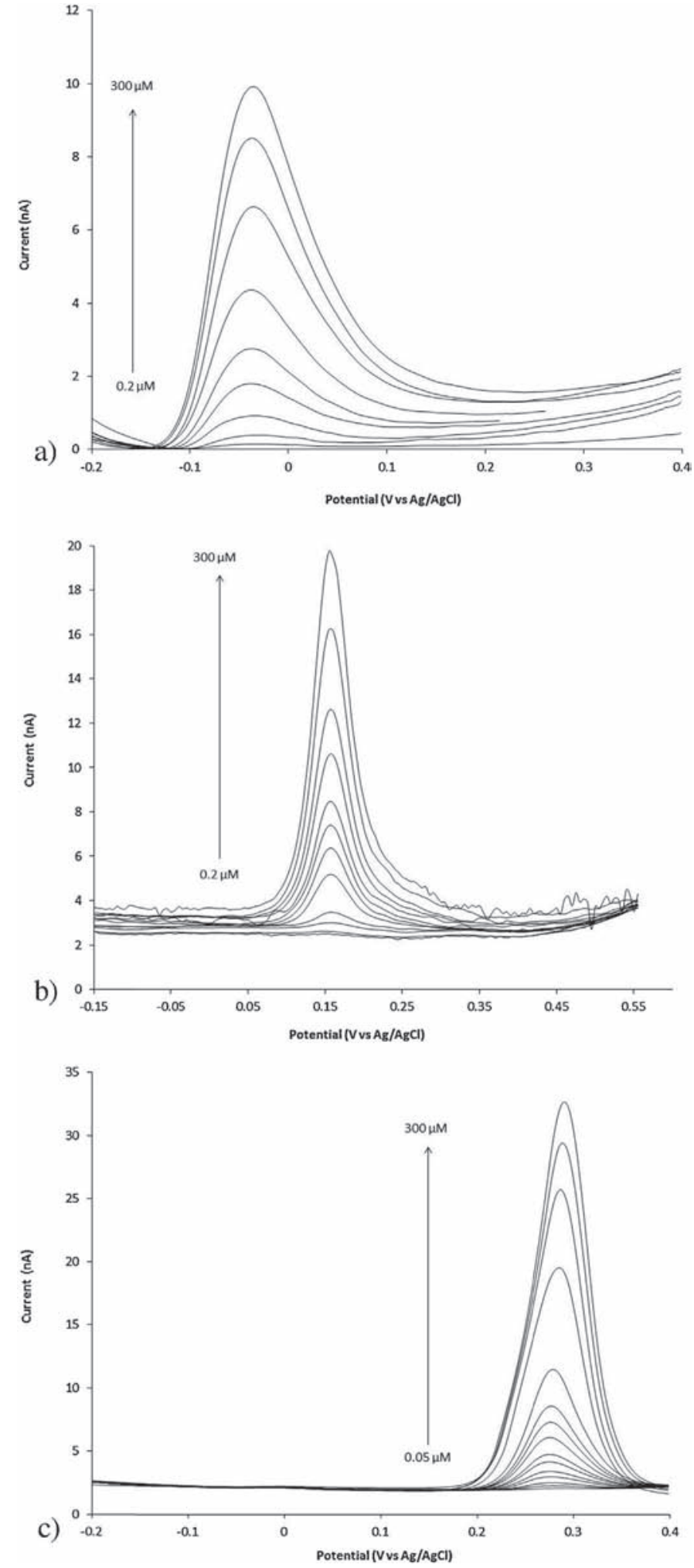

Fig. 7. Differential pulse voltammograms of (Au/PEDOT-Pt-Ag/AgCl) elecCell in $0.1 \mathrm{M}$ PBS ( $\mathrm{pH}$ 7.0) containing different concentrations of (a) ascorbic acid, (b) dopamine and (c) uric acid.

electrodes (Table 1), although analytes were used in excess for most of them. Finally, with the integrated electrochemical microdevice, it appears that a significant improvement in limits of detection was obtained compared to our previous results [39], making it more suitable for biological analysis. 


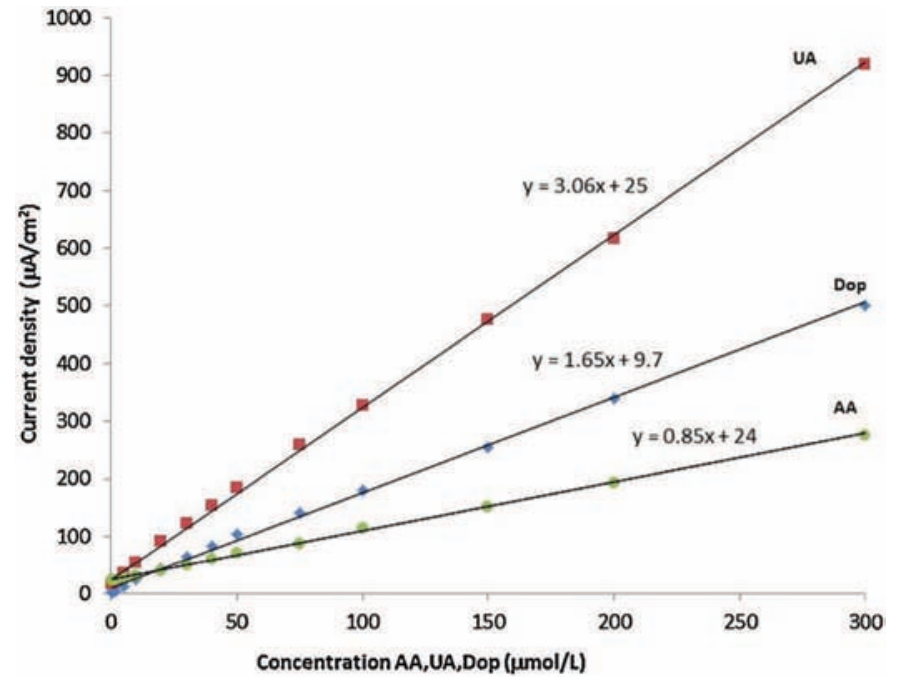

Fig. 8. Calibration curves for the three analytes: ascorbic acid, dopamine and uric acid.

\subsection{Reproducibility and stability}

The reproducibility and stability of the sensor were investigated by sensing studies. Ternary mixture of an equimolar solution of AA, Dop and UA $100 \mu \mathrm{M}$ was used for the reproducible examinations of five different $(\mathrm{Au} / \mathrm{PEDOT}-\mathrm{Pt}-\mathrm{Ag} / \mathrm{AgCl})$ ElecCell. The relative standard deviation (RSD) was found to be lower than $4.2 \%$ for AA, 4.5\% for Dop and 3.2\% for UA, suggesting that the ElecCell technology reproducibility was sufficiently good to deal with calibration. The stability of our sensors was examined in ternary mixture after being stored two weeks in air or in phosphate buffer solution (PBS). Thus, PEDOT modified microdevices retained 90\% of their initial sensitivities to the different antioxidant species studies (data not shown).

\section{Conclusion}

We have developed fully integrated, PEDOT-based, electrochemical microcells (ElecCell) allowing the selective detection of ascorbic acid, dopamine and uric acid in aqueous media. PEDOT has been successfully synthesized on integrated gold and platinum microelectrodes while using water and acetonitrile as solvent. According to DPV characterization, results show improved detection performances in term of sensitivity and selectivity for electrodeposited PEDOT layers, emphasizing good results using water as solvent, better results using acetonitrile as solvent and best results on gold surfaces compared to platinum ones. For this last and best case, detection properties of ascorbic acid, dopamine and uric acid were studied, exhibiting well-separated oxidation phenomena (oxidation potential: $-0.04,0.15$ and $0.28 \mathrm{~V}$, respectively), linear current variations, high sensitivities $(0.85$, 1.65 and $3.06 \mu \mathrm{A} / \mu \mathrm{M} \mathrm{cm}^{2}$, respectively) and low detection limit $(0.2 \mu \mathrm{M}, 0.1 \mu \mathrm{M}$ and $0.05 \mu \mathrm{M}$, respectively). As a result, the ElecCell technological platform is adapted to the mass fabrication of PEDOTmodified electrochemical devices for the analysis of antioxidant species. It was applied to model solutions up to now, but should be extended to real samples of blood sera and/or urines in the frame of clinical diagnosis and/or environmental applications.

\section{Acknowledgements}

The authors would like to thank Professor Maurice Comtat (LGC, Toulouse) for helpful discussions and advices. The technological realizations and associated research works were partly supported by the French RENATECH network.

\section{References}

[1] V. Beni, D.W.M. Arrigan, Microelectrode arrays and microfabricated devices in electrochemical stripping analysis, Curr. Anal. Chem. 4 (2008) 229-241.

[2] O.A. Sadik, A.O. Aluoch, A. Zhou, Status of biomolecular recognition using electrochemical techniques, Biosens. Bioelectron. 24 (2009) 2749-2765.

[3] J.W. Schultze, V. Tsakova, Electrochemical microsystem technologies: from fundamental research to technical systems, Electrochim. Acta 44 (1999) 3605-3627.

[4] C. Amatore, S. Arbault, C. Bouton, K. Coffi, J.C. Drapier, H. Ghandour, Y. Tong, Monitoring in real time with a microelectrode the release of reactive oxygen and nitrogen species by a single macrophage stimulation by its membrane mechanical depolarization, ChemBioChem 7 (2006) 653-661.

[5] A. Ruffien-Ciszak, P. Gros, M. Comtat, A.M. Schmitt, E. Questel, C. Casas, D. Redoules, Exploration of the global antioxidant capacity of the stratum corneum by cyclic voltammetry, J. Pharm. Biomed. Analysis 40 (2006) $162-167$.

[6] J.G. Roberts, J.V. Toups, E. Eyualem, G.S. McCarty, L.A. Sombers, In situ electrode calibration strategy for voltammetric measurements in vivo, Anal. Chem. 85 (2013) 11568-11575.

[7] R.S. Pai, K.M. Walsh, M.M. Crain, T.J. Roussel, D.J. Jackson, R.P. Baldwin, R.S. Keynton, J.F. Naber, Fully integrated three-dimensional electrodes for electrochemical detection in microchips: fabrication, characterization and applications, Anal. Chem. 81 (2009) 4762-4769.

[8] Y.P. Chen, Y. Zhao, J. Chu, S.Y. Liu, W.W. Li, G. Liu, Y.C. Tian, Y. Xiong, H.Q. Yu, Fabrication and characterization of an innovative solid-state microelectrode, Electrochim. Acta 55 (2010) 5984-5989.

[9] K. Dawson, A. Wahl, S. Barry, C. Barrett, N. Sassiat, Fully-integrated on-chip nano-electrochemical devices for electroanalytical applications, Electrochim. Acta 115 (2014) 239-246.

[10] N. Pereira-Rodriguez, Y. Sakai, T. Fujii, Cell-based microfluidic biochip for the electrochemical real-time monitoring of glucose and oxygen, Sens. Actuators B 132 (2008) 608-613.

[11] M. Miyashita, N. Ito, S. Ikeda, T. Murayama, K. Oguma, J. Kimura, Development of urine glucose meter based on micro-planar amperometric biosensor and its clinical application for self-monitoring of urine glucose, Biosens. Bioelectron. 24 (2009) 1336-1340.

[12] S. Ben Amor, E. Vanhove, F. Sékli Belaïdi, S. Charlot, D. Colin, M. Rigoulet, A Devin, J. Launay, P. Temple-Boyer, S. Arbault, Enhanced detection of hydrogen peroxide with platinized microelectrode arrays for analyses of mitochondria activities, Electrochim. Acta 126 (2014) 171-178.

[13] O. Frey, P.D. van der Wal, S. Spieth, O. Brett, K. Seidl, O. Paul, P. Ruther, R. Zengerle, N.F. de Rooij, Biosensor microprobes with integrated microfluidic channels for bi-directional neurochemical detection, J. Neural Eng. 8 (2011) $1-9$.

[14] A. Altuna, L. Menendez de la Prida, E. Bellistri, G. Gabriel, A. Guilera, J. Berganzo, R. Vila, L.J. Fernandez, SU-8 based microprobes with integrated planar electrodes for enhanced neural depth recording, Biosens. Bioelectron. 37 (2012) $1-5$.

[15] J. Dawson, P. Jeemon, L. Hetherington, C. Judd, C. Hastie, C. Schulz, W. Sloan, S. Muir, A. Jardine, G. McInnes, D. Morrison, A.F. Dominiczak, S. Padmanabhan, M. Walters, Serum uric acid level, longitudinal blood pressure, renal function, and long-term mortality in treated hypertensive patients, Hypertension 62 (2013) $105-111$.

[16] S.S. Rodriguez, K.A. Salazar, N.A. Jara, M.A. Garcia-Robles, F. Perez, L.E. Ferrada, E. Luciano, F. Martinez, F.J. Nualart, Superoxide-dependent uptake of vitamin C in human glioma cells, J. Neurochem. 127 (2013) 793-804.

[17] S.D. Cekic, A. Cetinkaya, A.N. Avan, R. Apak, Correlation of total antioxidant capacity with reactive oxygen species (ROS) consumption measured by oxidative conversion, J. Agric. Food Chem. 61 (2013) 5260-5270.

[18] O. Orrigoni, M.C. De Tullio, Ascorbic acid: much more than just an antioxidant, Biochim. Biophys. Acta 1569 (2002) 1-9.

[19] R.M. Wightman, L.J. May, A.C. Michael, Detection of dopamine dynamics in the brain, Anal. Chem. 60 (1988) 769A-793A.

[20] C. Martin, The Parkinson's puzzle: new developments in our understanding of Parkinson's disease have generated a number of promising new treatments for this disabling condition, Chem. Britain 34 (1998) 40-42.

[21] V.V.S. Eswara Dutt, H.A. Mottola, Determination of uric acid at the microgram level by a kinetic procedure based on a pseudo-induction period, Anal. Chem. 46 (1974) 1777-1781

[22] H. Etnet, M. Knoll, Electrochemical characterization of uric acid and ascorbic acid at a platinum electrode, Anal. Chim. Acta 449 (2001) 129-134.

[23] C. Christophe, F. Sékli Belaïdi, J. Launay, P. Gros, E. Questel, P. Temple-Boyer, Elaboration of integrated microelectrodes for the detection of antioxidant species, Sens. Actuators B77 (2013) 350-356.

[24] M. Roushani, M. Shamsipur, H.R. Rajabi, Highly selective detection of dopamine in the presence of ascorbic acid and uric acid using thioglycolic acid capped CdTe quantum dots modified electrode, J. Electroanal. Chem. 712 (2014) $19-24$. 
[25] B. Kaur, T. Pandiyan, B. Satpati, R. Srivastava, Simultaneous and sensitive determination of ascorbic acid, dopamine, uric acid, and tryptophan with silver nanoparticules-decorated reduced graphene oxide modified electrode, Colloid Surf. B 111 (2013) 97-106

[26] X. Wang, M. Wu, W. Tang, Y. Zhu, L. Wang, P. He, Y. Fang, Simultaneous electrochemical determination of ascorbic acid, dopamine and uric acid using a palladium nanoparticle/graphene/chitosan modified electrode, J. Electroanal. Chem. 695 (2013) 10-16.

[27] T.Q. Xu, Q.L. Fhang, J.N. Zheng, Z.Y. Lv, J. Wei, A.J. Wang, J.J. Feng, Simultaneous determination of dopamine and uric acid in the presence of ascorbic acid using Pt nanoparticles supported on reduced graphene oxide, Electrochim. Acta 115 (2014) 109-115.

[28] E. de Pieri Troiani, E.R. Pereira-Filho, R. Censi Faria, Chemometric strategies to develop a nanocomposite electrode for simultaneous determination of ascorbic acid, dopamine, and uric acid, Electroanalysis 23 (2011) 2822-2831.

[29] H. Li, Y. Wang, D. Ye, J. Luo, B. Su, S. Zhang, J. Kong, An electrochemical sensor for simultaneously determination of ascorbic acid, dopamine, uric acid and tryptophan based on MWNTs bridged mesocellular graphene foam nanocomposite, Talanta 127 (2014) 255-261.

[30] J.Zhan, Z. Zhu, J.Zhu, K. Li, S. Hua, Selective determination of dopamine, ascorbic acid and uric acid at SDS-MWCNTs modified glassy carbon electrode, Int. J. Electrochem. Soc. 9 (2014) 1264-1272.

[31] P. Manivel, M. Dhakshnamoorthy, A. Balamurugan, N. Ponpandian, D. Mangalaraj, C. Viswanathan, Conducting polyaniline-graphene oxide fibrous nanocomposites: preparation, characterization and simultaneous electrochemical detection of ascorbic acid, dopamine and uric acid, RSC Adv. 3 (2013) $14428-14437$.

[32] J. Du, R. Yue, F. Ren, Z. Yao, F. Jiang, P. Yang, Y. Du, Novel graphene flowers modified carbon fibers for simultaneous determination of ascorbic acid, dopamine and uric acid, Biosens. Bioelectron. 53 (2014) 220-224

33] D. Wu, Y. Li, Y. Zhang, P. Wang, Q. Wei, B. Du, Sensitive electrochemical sensor for simultaneous determination of dopamine, ascorbic acid, and uric acid enhanced by amino-group functionalized mesoporous $\mathrm{Fe}_{3} \mathrm{O}_{4} @$ graphene sheets, Electrochim. Acta 116 (2014) 244-249.

[34] Y. Li, X. Lin, Simultaneous electroanalysis of dopamine, ascorbic acid and uric acid by poly (vinyl alcohol) covalently modified glassy carbon electrode, Sens. Actuators B 115 (2006) 134-139.

[35] M. Mazloum-Ardakani, M.A. Sheikh-Mohseni, A. Benvidi, Electropolymerization of thin film conducting polymer and its application for simultaneous determination of ascorbic acid, dopamine and uric acid, Electroanalysis 23 (2011) 2822-2831.

[36] J. Samseya, R. Srinivasan, Y.T. Chang, C.W. Tsao, V.S. Vasantha, Fabrication and characterisation of high performance polypyrrole modified microarray sensor for ascorbic acid determination, Anal. Chim. Acta 793 (2013) $11-18$

[37] S.S. Kumar, J. Mathiyarasu, K.L.N. Phani, V. Yegnaraman, Simultaneous determination of dopamine and ascorbic acid on poly (3 4-ethylenedioxythiophene) modified glassy carbon electrode, J. Solid State Electrochem. 10 (2006) 905-913.

[38] J. Mathiyarasu, S. Senthilkumar, K.L.N. Phani, V. Yegnaraman, PEDOT-Au nanocomposite film for electrochemical sensing, Mater. Lett. 62 (2008) $571-573$.

[39] F. Sekli Belaidi, P. Temple-Boyer, P. Gros, Voltammetric microsensor using PEDOT modified gold electrode for the simultaneous assay of ascorbic and uric acids, J. Electroanal. Chem. 647 (2010) 159-168.

[40] K.C. Lin, C.Y. Yin, S.M. Chen, Simultaneous determination of AA, DA and UA based on bipolymers by electropolymerization of luminol and 3,4-ethylenedioxythiophene monomers, Int. J. Electrochem. Sci. 6 (2011) 3951-3965.

[41] S. Yu, C. Luo, L. Wang, H. Peng, Z. Zhu, Poly (3 4-ethylenedioxythiophene)modified $\mathrm{Ni} /$ silicon microchannel plate electrode for the simultaneous determination of ascorbic acid, dopamine and uric acid, Analyst 138 (2013) 1149-1155.

[42] K.C. Lin, J.Y. Huang, S.M. Chen, Simultaneous determination of ascorbic acid dopamine, uric acid and hydrogen peroxide based on co-immobilization of PEDOT and FAD using multi-walled carbon nanotubes, Anal. Methods 6 (2014) 8321-8327.

[43] L.B. Groenendaal, F. Jonas, D. Freitag, H. Pielartzik, J.R. Reynolds, Poly(3,4ethylenedioxythiophene) and its derivatives: past, present, and future, Adv. Mater. 12 (2000) 481-494.

[44] R. Jolly, S. Pairis, C. Petrescu, Comparative ageing of three electroconductive polymers, J. Chim. Phys. 95 (1998) 1400-1405.

[45] K. Lerch, F. Jonas, M. Linke, Properties and applications of Baytron (PEDT), J. Chim. Phys. 95 (1998) 1506-1509.

[46] J. Roncali, A. Yassar, F. Garnier, Electrosynthesis of highly conducting poly(3methylthiophene) thin films, J. Chem. Soc. Chem. Commun. 9 (1988) 581-582.

[47] C.P. Andrieux, J.M. Dumas-Bouchiat, J.M. Savéant, Kinetics of electrochemical reactions mediated by redox polymers films, J. Electranal. Chem. 169 (1984) 9-21.

[48] E. Vanhove, A. Tsopéla, L. Bouscayrol, A. Desmoulin, J. Launay, P. Temple-Boyer, Final capping passivation layers for long-life microsensors in real fluids, Sens. Actuators B 178 (2013) 350-358

[49] V. Castagnola, C. Bayon, E. Descamps, C. Bergaud, Morphology and conductivity of PEDOT layers produced by different electrochemical routes, Synth. Metals 189 (2014) 7-16.
[50] E. Tamburri, S. Orlanducci, F. Toschi, M.L. Terranova, D. Passeri, Growth mechanisms, morphology and electroactivity of PEDOT layers produced by different electrochemical routes in aqueous medium, Synth. Metals 159 (2009) 406-414.

[51] J. Bobacka, A. Lewenstam, A. Ivaska, Electrochemical impedance spectroscopy of oxidized poly (3 4-ethylenedioxythiophene) film electrodes in aqueous solutions, J. Electroanal. Chem. 489 (2000) 17-27.

[52] L. Pigani, B. Zanfrognini, R. Seeber, PEDOT-modified microelectrodes, preparation, characterisation and analytical performances, Electroanalysis 24 (2012) 1340-1347.

[53] J.C. Gustafsson, B. Ledberg, O. Inganäs, In situ spectroscopic investigations of electrochromism and ion transport in a poly (3 4-ethylendioxythiophene) electrode in a solid state electrochemical cell, Solid State Ionics 69 (1994) $145-152$.

[54] T.F. Otero, I. Cantero, H. Grande, Solvent effects on the charge storage ability in polypyrrole, Electrochim. Acta 44 (1999) 2053-2059.

[55] R. Kiefer, G.A. Bowmaker, R.P. Cooney, P.A. Kilmartin, J. Travas-Sejdic, Cation driven actuation for free standing PEDOT films prepared from propylene carbonate electrolytes containing $\mathrm{TBACF}_{3} \mathrm{SO}_{3}$, Electrochim. Acta 53 (2008) 2593-2599.

[56] H.J. Ahonen, J. Lukkari, T. Hellstrom, J. Mattila, J. Kankare, Characterisation of poly (34-ethylenedioxythiophene) films polymerised in aqueous media, Synth. Metals 119 (2001) 119-120.

[57] S. Patra, K. Parai, N. Munichandraiah, Scanning electron microscopy studies of PEDOT prepared by various electrochemical routes, Synth. Metals 158 (2008) 430-435.

[58] G.E. Poirier, M.J. Tarlov, Molecular ordering and gold migration observed in butanethiol self-assembled monolayers using scanning tunnelling microscopy, J. Phys. Chem. A 99 (1995) 10966-10970.

[59] Y.C. Yang, Y.P. Yen, L.Y. Yan, S.L. Yau, K. Itaya, Elucidation of the deposition processes and spatial structures of alkanethiol and arylthiol molecules adsorbed on Pt(111) electrodes with in situ scanning tunnelling microscopy, Langmuir 20 (2004) 10030-10037.

[60] P. Boulanger, J., Polonovski, F., Tayeau, P. Mandel and G. Biserte, Biochimie Médicale, 8th Edition, Masson, Paris, 1971.

[61] M.C. Polidori, W. Stahl, O. Eichler, I. Niestroj, H. Sies, Profiles of antioxidants in human plasma, Free Radic. Biol. Med. 30 (2001) 456-462.

\section{Biographies}

Fadhila Sekli Belaïdi was born on February 22 1980. She received her Master's Degree in process and environmental engineering from the "Institut National des Sciences Appliquées de Toulouse" (France) in 2006. She joined the "Laboratoire de Génie Chimique" (LGC) from the University of Toulouse (France) in 2007. She is working on the development of electrochemical microsensors for chemical and biochemical detection.

Aurélie Civélas was born in Aix-en-Provence, France, on January 12 1989. She joined the "Laboratoire d'Analyse et d'Architecture des Systèmes" (LAAS) of the "Centre National de la Recherche Scientifique" (CNRS) of Toulouse in 2012 for a one year training course. She worked on the development of electrochemical microsensors for chemical and biochemical detection. She received the degree in electronic Engineering from the in "Chimie - Physique-Electronique" school (Lyon-France) in 2014

Valentina Castagnola was born in Bologna, Italy in 1986. She received the master degree in photochemistry and material chemistry from the University of Bologna, in 2011. She joined the "Laboratoire d'Analyse et d'Architecture des Systèmes" of the "Centre National de la Recherche Scientifique" (LAAS-CNRS), in 2011 as PhD Student. She is carrying out her experimental research concerning implantable microdevices for the recording of the neural activity.

A. Tsopela was born in Athens, Greece in 1988. She received the master degree in chemical engineering from the National Technical University of Athens (NTUA Greece), in 2011. She joined the "Laboratoire d'Analyse et d'Architecture des Systèmes" of the "Centre National de la Recherche Scientifique" (LAAS-CNRS), in 2011 as PhD Student. She is carrying out her experimental research in the development of microsensors with environmental applications.

Laurent Mazenq was born on May 30,1982. He joined the Laboratoire d'Architecture et d'Analyse des Systèmes of the French Centre National de la Recherche Scientifique (LAAS-CNRS) in 2002. Since then, he has been working on photolithography and on micro/nanotechnologies process realization.

Pierre Gros was born in 1970. He graduated in physical chemistry in 1992 and received his PhD degree in Chemical Engineering in 1996 at the University Paul Sabatier in Toulouse. He is now Professor in Electroanalytical Engineering in the Chemical Engineering Laboratory (Toulouse-France). He is currently working on the development of electrochemical (bio)sensors.

Jérôme Launay was born on March 11, 1975. He received the degree in electronic engineering from the Institut National des Sciences Appliquées de Toulouse" (France) in 1998. He joined the "Laboratoire d'Analyse et d'Architecture des Systèmes" from the French "Centre National de la Recherche Scientifique" (LAAS-CNRS) in 1998 and received the $\mathrm{PhD}$ degree from the "Institut National des Sciences Appliquées de Toulouse" (France) in 2001. In 2002, he became lecturer at the 
University of Toulouse (France). His research activities include the development of electrochemical microsensors for the detection in liquid phase.

Pierre Temple-Boyer was born on October 25, 1966. He received his Engineer's Degree in electronic engineering from the "Ecole Supérieure d'Electricité" (Paris-France) in 1990 and his Master's Degree in microelectronics from the
University of Toulouse (France) in 1992. He joined the "Laboratoire d'Analyse et d'Architecture des Systèmes" (LAAS) from the French "Centre National de la Recherche Scientifique" (CNRS) in 1992 and received the PhD degree from the "Institut National des Sciences Appliquées de Toulouse" (France) in 1995. Since then, as a CNRS researcher, he has worked at LAAS on the development of physical and chemical microsensors. 